\title{
Protein-Pd Complexes Conjugates as Artificial Enzymes
}

\author{
Jose M Palomo ${ }^{\star, a}$ and Esteban P. Urriolabeitia ${ }^{\star, b}$ \\ ${ }^{a}$ Department of Biocatalysis, Institute of Catalysis (CSIC)Marie Curie 2. Cantoblanco. Campus UAM, \\ 28049Madrid, Spain \\ ${ }^{b}$ Instituto de Síntesis Quimica y Catálisis Homogénea (ISQCH, CSIC-Universidad de Zaragoza), \\ C/Pedro Cerbuna 12, Zaragoza, E-50009, Spain \\ Email: josempalomo@icp.csic.es (J. M. P.), esteban@unizar.es (E. P. U.)
}

\begin{abstract}
The combination of enzymes and palladium organometallic complexes has been recently developed for the creation of new types of artificial metalloenzymes. Different methods considering the kind of protein used, from a core protein to an enzyme, or the strategies for the selective insertion of the organometallic complex from direct Pd bonding to protein amino acid, affinity interactions or covalent attachment by a Pd complex ligand have been developed. This methodology affords new Pd catalysts with highest selectivity and activity in different reactions at milder conditions compared with the free $\mathrm{Pd}$ complexes
\end{abstract}

Keywords metalloenzymes, palladium complexes, hybrids, organometallics

Palladium complexes have shown a great relevance in different areas, especially in catalytic application in different process such as $\mathrm{C}-\mathrm{C}$ bond formation, $\mathrm{C}-\mathrm{H}$ activation, reduction, multicomponent processes, ${ }^{[1,2]}$ but also in biomedical applications ${ }^{[3]}$ and as GFP-like fluorophores. ${ }^{[4,5]}$

Metalloenzymes are natural biological catalysts. They contain a metal nucleus involved in their catalytic activity in many important biological processes. ${ }^{[6]}$

However, in many cases, these enzymes have a specific activity towards a given reaction. In this way, the design of artificial metalloenzymes combining organometallic complexes with proteins or enzymes has allowed the development of enzymes with synthetic activity in an elegant way. ${ }^{[7,8]}$

In particular, considering the advantages of $\mathrm{Pd}$ as catalyst, the development of new Pd-enzymes will be challenging. The three-dimensional structure of proteins offers a second coordination sphere, which is critical for high selectivity processes. This combined with tailor-made Pd organometallics have been successfully applied for the development of a new kind of synthetic artificial enzymes (Figure 1). ${ }^{[9-12]}$ In addition, the combination with the protein makes possible to take advantage of the mild enzymatic reaction conditions for performing more sustainable palladium chemistry.
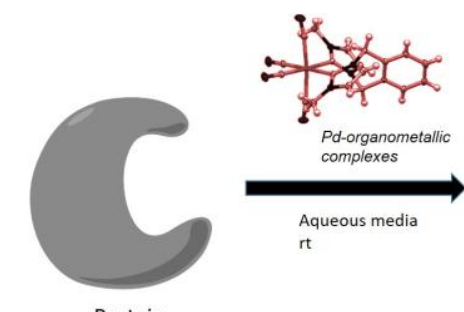

Protein
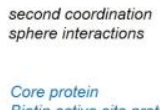

Biotin active site protein
Enzyme active site

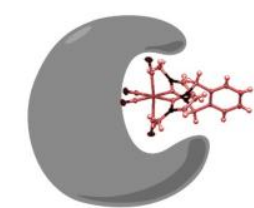

Pd organometallicsprotein conjugates

Insertion methods: Pd-protein coordination High affinity moleculeprotein Ligand-protein coordination

Figure 1 Concept of the preparation of artificial Pd-enzymes.
Within the methods for the preparation of these artificial Pd enzymes, there are not many examples of such conjugates so far. In the preparation of these bioconjugates, the use of a core protein (human serum albumin (HSA), ferritin), the avidin-biotin strategy or, directly, the use of an enzyme with hydrolytic activity transformed into synthetic activity have been employed as synthetic strategies. ${ }^{[9-12]}$

In addition to the type of anchoring either in a protein without catalytic activity or the use of a cavity with catalytic capability, the metal insertion method has also an important influence.

There are synthetic alternatives, where the insertion of the metal is carried out through the modification of a molecule. For instance, the biotin takes advantage of its high affinity with avidin or streptavidin, incorporating in a defined area of the structure. ${ }^{[10]}$ The application of protein genetic modification strategies have allowed the alteration of catalytic cavity to improving $\mathrm{Pd}$ complexes selectivity in $\mathrm{C}-\mathrm{C}$ bond forming reactions. ${ }^{[10]}$

Another strategy lies in the insertion of the organometallic complex directly through one of its ligands. This case is represented by the elegant strategy of inserting a $\mathrm{Pd}$ pincer into the active site of a hydrolase.$^{[9]}$ In that case, the synthesis used a ligand that mimics the structure of irreversible inhibitors of these enzymes through phospho-derivatives. In this example, the modification was also carried out in solid phase, where the enzyme was previously immobilized. This showed that insertion of the complex is possible in this way and allowed the preparation, for the first time, of a heterogeneous metalloenzyme with synthetic activity towards the Heck reaction, which also proved to be completely enantioselective. ${ }^{[9]}$ The presence of the enzyme also allows to perform the reaction at milder conditions with the conjugates than with the free complex.

This technology also allows the synthesis of a Pd-metalloenzyme with high activity and excellent recyclability.

A third option is the use of a protein cage, such as apo-ferritin, as core for insertion of allyl-Pd complexes via multinuclear metal complexes. Those complexes show various coordination structures, prepared by the deletion or introduction 


\section{Perspective}

of key amino acid residues at appropriate positions of the protein. ${ }^{[11]}$

Although there are examples that show the great potential of this technology in palladium chemistry, future lines of research would be focused on the application of robust enzymes that allow us to extend the synthetic variability of $\mathrm{Pd}$ complexes under more drastic conditions. Additional challenges comprise the development of protein modification strategies in the environment of an enzyme, allowing the anchoring of the metal to the protein directly or through specific ligands. In this respect, the insertion of a selective reactive group in the protein, by chemical or genetic methods, would allow to evaluate the effect of the arrangement of the complex on the protein environment and the effect that this could cause on its properties.

Future lines of development in this regard, combined with the use of solid phase strategies, as well as the use of alternative protein sources that do not economically imply a ballast, would allow artificial Pd-enzymes to be achieved in sufficient quantity for a possible real implementation of these systems.

\section{Acknowledgements}

We want to thank to the Spanish National Research Council (CSIC, Spain), the Aragon Government (Spain, Project LMA188_14) and the European Cooperation in Science and Technology (COST) program under CA15106 grant (CHAOS: $\mathrm{CH}$ Activation in Organic Synthesis).

\section{References}

[1] Biffis, A.; Centomo, P.; Del Zotto, A.; Zecca, M. Chem. Rev. 2018, 1184, 2249.

[2] Gensch, T.; Hopkinson, M. N.; Glorius, F.; Wencel-Delord, J. Chem. Soc. Rev. 2016, 45, 2900.

[3] Kapdi, A. R.; Fairlamb, I. J. S. Chem. Soc. Rev. 2014, 43, 4751.

[4] Carrera, C.; Denisi, A.; Cativiela, C.; Urriolabeitia, E. P. Eur. J. Inorg. Chem. 2019, 2019, 3481.

[5] Collado, S.; Pueyo, A.; Baudequin, C.; Bischoff, L.; Jiménez, A. I.; Cativiela, C.; Hoarau, C.; Urriolabeitia, E. P. Eur. J. Org. Chem. 2018, 2018, 6158.

[6] Schilter, D. Nat. Rev. Chem. 2017, 1, 0081.

[7] Lewis, J. C. Acc. Chem. Res. 2019, 52, 576.

[8] Artificial Metalloenzymes and MetalloDNAzymes in Catalysis: From Design to Applications. ISBN: 978-3-527-80409-2. Ed.: Diéguez, M.; Bäckvall, J.-E.; Pàmies, O. 2018, p. 1200.

[9] Filice, M.; Romero O.; Aires, A.; Guisan, J. M.; Rumbero, A.; Palomo, J. M. Adv. Synth. Catal. 2015, 357, 2687.

[10] Chatterjee, A.; Mallin, H.; Klehr, J.; Vallapurackal, J.; Finke, A. D.; Vera, L.; Marsh, M.; Ward, T. R. Chem. Sci. 2016, 7, 673.

[11] Abe, S.; Niemeyer, J.; Abe, M.; Takezawa, Y.; Ueno, T.; Hikage, T.; Erker, G.; Watanabe, Y. J. Am. Chem. Soc. 2008, 130, 10512.

[12] Pocquet, P.; Vologdin, N.; Mangiatordi, G. F.; Ciofini, I.; Nicolotti, O.; Thorimbert, S.; Salmain, M. Eur. J. Inorg. Chem. 2017, 2017, 3622.

Received November 5, 2019 Accepted November 28, 2019 